\title{
Utilizing Moving Vehicles as Sensors for Bridge Condition Screening-A Laboratory Verification
}

\author{
Chul-Woo Kim*, Kai-Chun Chang, Patrick John McGetrick, ${ }^{1}$ \\ Shinichi Inoue, ${ }^{2}$ and Souichiro Hasegawa \\ Department of Civil and Earth Resource Engineering, Graduate School of Engineering, Kyoto University, \\ Kyotodaigaku-katsura, Nishikyo, Kyoto 615-8540, Japan \\ ${ }^{1}$ School of Natural and Built Environment, Queen's University Belfast, \\ David Keir Building, Stranmillis Road, Belfast, BT9 5AG, UK \\ ${ }^{2}$ Nikken Sekkei Civil Engineering Ltd., 1-4-27 Koraku, Bunkyo, Tokyo 112-0004, Japan
}

(Received July 9, 2016; accepted November 24, 2016)

Keywords: damage detection, drive-by method, spectral analysis, roadway profile identification, vibration-based structural health monitoring

Health condition monitoring of bridge structures is attracting considerable attention, conventionally relying on visual inspection, and measurement-based methods that involve sensors installed directly on bridges. In recent years, drive-by monitoring methods that treat moving vehicles as moving sensors have been proposed as alternatives; these methods aim to be lowcost, mobile, and target fast bridge condition screening. In this study, we address the current lack of sufficient experimental verification of such methods. Laboratory experiments were conducted using a test vehicle system equipped with accelerometers in order to verify the practical feasibility of three drive-by methods: (1) bridge-frequency extraction using the Fourier spectrum of a vehicle's dynamic response, (2) damage detection using the change in a vehicle's spectral distribution pattern, and (3) roadway surface profile identification.

\section{Introduction}

There is an increasing demand for the health monitoring of bridge structures all over the world. Taking Japan as an example, it was estimated statistically that the percentage of bridges having served over 50 years would reach $28 \%$ in 10 years, and would exceed $53 \%$ in 20 years. ${ }^{(1)}$ The traffic loadings on those bridges may have changed in recent decades, mostly increased, which might accelerate the deterioration of the aging bridges. To monitor the health condition of this significant number of aging bridges, as well as newly constructed bridges, resource allocation needs will continue to increase with the requirement for inspection tasks aimed at the detection of potential bridge damage. Moreover, a huge number of urgent inspection tasks might be necessary when a natural disaster such as an earthquake or tsunami strikes. Traditionally, bridge inspections have primarily relied on visual inspection methods that are limited to detecting visible damage, and they usually produce large variations in the inspection results. It is suggested that visual inspection alone may be inadequate for bridge health monitoring. ${ }^{(2)}$ Therefore, measurement-based monitoring and damage detection methods play increasingly important roles.

*Corresponding author: e-mail: kim.chulwoo.5u@kyoto-u.ac.jp http://dx.doi.org/10.18494/SAM.2017.1433 
A popular damage detection approach utilizes the vibration responses of target bridges, given the intuitive fact that changes in structural integrity are linked to changes in structural vibration responses and their dynamic properties. ${ }^{(3,4)}$ Conventionally, those vibration-based damage detection methods involve the deployment and maintenance of vibration sensors on target bridges, which is costly, time-consuming, and sometimes dangerous. In addition, the implementation of monitoring systems is not widespread for short- and medium-span bridges, which form the greatest proportion of bridges in service.

A complementary approach is to utilize the vibrations of a test vehicle, rather than the vibrations of the bridge, when the vehicle passes over the target bridge. ${ }^{(5)}$ It involves a test vehicle instrumented with sensors, the responses of which are used to extract the dynamic properties of the target bridge. Through the interaction between the vehicle and the bridge, the test vehicle can be considered to have a dual role as both exciter and receiver of the bridge vibrations. For convenience, the measurement methods that involve mounting sensors directly on the bridge body are referred to as direct methods, while those that involve mounting sensors on the test vehicle are referred to as indirect methods ${ }^{(6)}$ or drive-by methods. ${ }^{(7)}$ The drive-by inspection methods are considered low-cost, mobile, and target fast bridge condition screenings; it follows that such methods also reduce the need for the installation and maintenance of a sensor network directly on the bridge.

Over the past decade, many new drive-by methods have been proposed, initially focusing on bridge-frequency extraction ${ }^{(5)}$ and later expanding to damage detection, ${ }^{\left({ }^{(}\right)}$roadway surface profile identification, ${ }^{(9)}$ and a range of bridge monitoring techniques. ${ }^{(10)}$ However, most of the studies have been presented analytically and numerically; few studies have focused on the practical verification of these methods in laboratories ${ }^{(7,11)}$ and field trials. ${ }^{(6,12)}$ Therefore, their practical applicability is still an open question.

The primary aim of this study is to verify the feasibility of utilizing moving vehicles as moving sensors in terms of the drive-by bridge inspection via scaled laboratory experiments. Three existing methods were tested here: (1) bridge-frequency extraction using the Fourier spectrum of a vehicle's dynamic response, (2) damage detection using the change in a vehicle's spectral distribution pattern, and (3) roadway surface profile identification. The scaled bridge model, experimental vehicle system, and instrumentation are first described, and then the theoretical background and experimental results for the three methods are presented. Finally, several concluding remarks are drawn regarding their practical applicability.

\section{Laboratory Experiment}

\subsection{Experiment bridge model}

The experiment setup and roadway roughness profile used in the laboratory experiment are shown in Fig. 1. The scaled experiment bridge comprised three spans of simply supported beams: the main target span at the center that was preceded by an accelerating span and followed by a decelerating span. The main span was a steel I girder of $5.4 \mathrm{~m}$ span length. A pair of rails was attached to the top surface of the girder, serving as the track along which the inspection vehicle can move. The rail surfaces were laser-engraved with a set of roughness profiles that resembled corresponding profiles measured on a real roadway. The profiles were scaled down proportionally from a path of $40.4 \mathrm{~m}$ long in the longitudinal direction and scaled down with a ratio of $1 / 10$ in the vertical direction. 

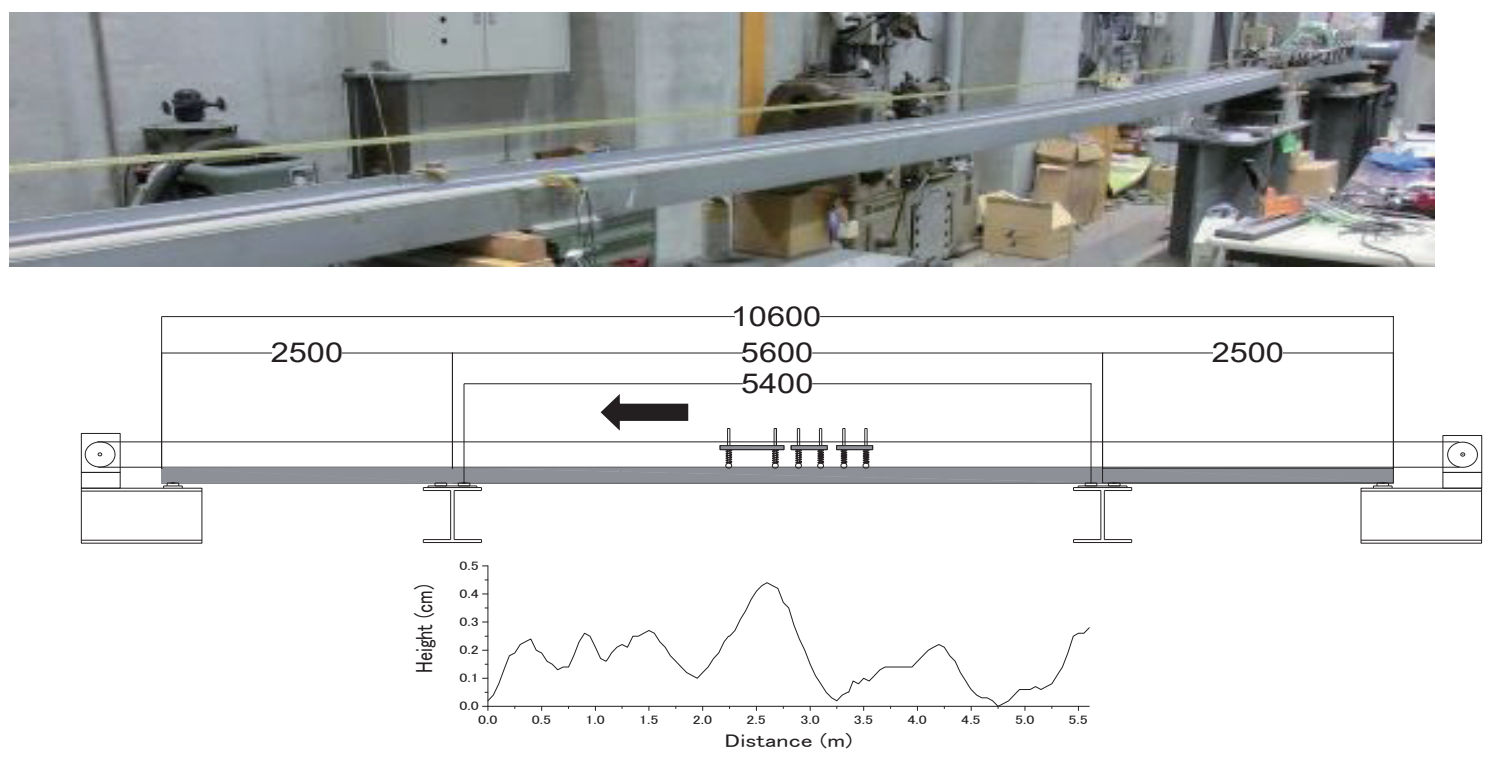

Fig. 1. (Color online) Scaled bridge model and surface roughness profile.

Uniaxial accelerometers (AS-1GB model by Kyowa Electronic Instruments Co.; range, \pm 9.8 $\mathrm{m} / \mathrm{s}^{2}$; resonance frequency, $70 \mathrm{~Hz}$ ) were deployed at the quarter-span, mid-span, and three-quarterspan to measure the bridge's vertical acceleration responses. The dynamic properties of the bridge were obtained from an independent free vibration test and are listed in Table 1.

\subsection{Experimental vehicle system}

The experimental vehicle system comprised three vehicles: one tractor and two trailers, as shown in Fig. 2. The tractor was a two-axle vehicle, which was heavier than the trailers and served to excite the bridge into vibration. The trailers were two-axle vehicles, which would be excited by the bridge that was already in vibration and hence primarily served as receivers of the bridge vibrations. The two trailers were used in order to eliminate or reduce the unwanted roadway profileinduced vibration components and consequently let the bridge-related vibration components stand out. Two uniaxial accelerometers (AS-1GB model) were mounted on each trailer, one on the front and the other on the rear axle, to measure the trailers' vertical vibrations. These accelerometers were linked to a dynamic data recorder (DC-104R model by Tokyo Sokki Kenkyujo Co.) installed on the tractor; the data recorder was remotely controlled and monitored by a manual control panel via wireless transmission. The wireless transmission was realized using an $11 \mathrm{~g} \& \mathrm{~b}$ wireless LAN router (WHR-HP-G model by Buffalo Inc.).

The two trailers were assembled with identical components and configurations so as to behave with similar dynamic characteristics. The dynamic properties of the tractor and trailers were measured in an independent free vibration test and are given in Table 2. The total mass of the vehicle system was $33 \mathrm{~kg}$, about $1 / 9$ of that of the test bridge $(297 \mathrm{~kg})$. For all measurements, the sampling frequency was set as $100 \mathrm{~Hz}$. The vehicle speed was controlled by an external motor and kept constant. Three constant speeds were tested in this study: $S 1=0.55 \mathrm{~m} / \mathrm{s}, S 2=1.05 \mathrm{~m} / \mathrm{s}$, and $S 3=2.45 \mathrm{~m} / \mathrm{s}$. There were optical sensors installed at both ends of the test bridge span to detect the entrance and exit of the experimental vehicle. These optical sensors were PZ-G52NR models by Keyence Co. 
Table 1

Bridge dynamic properties.

1st natural frequency $\quad 3.64 \mathrm{~Hz}$

2nd natural frequency $14.45 \mathrm{~Hz}$

Damping ratio 0.011

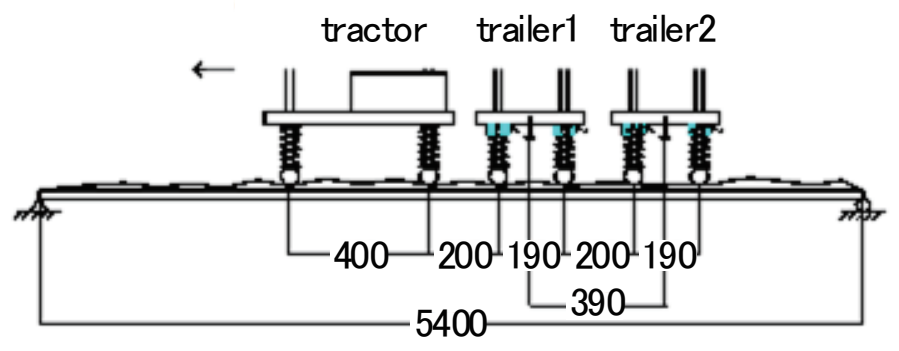

(a)

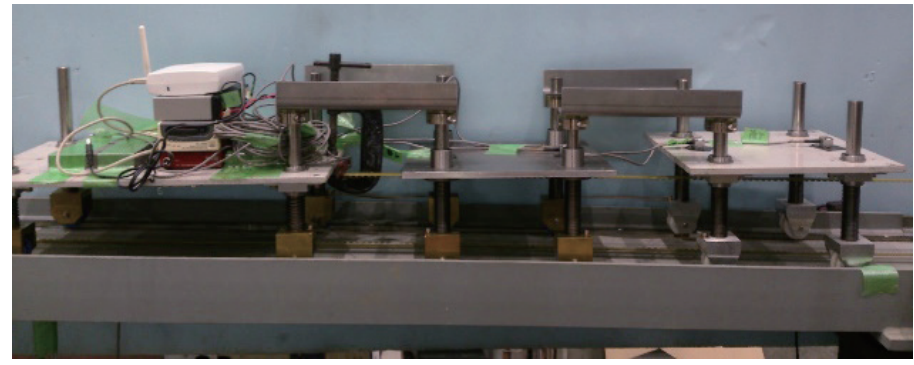

(b)

Fig. 2. (Color online) Tractor-trailer system: (a) drawing with sensor layout (marked in blue) (unit: mm) and (b) photo.

Table 2

Vehicle dynamic properties.

\begin{tabular}{lcc}
\hline & Tractor & Trailer \\
\hline Total mass $(\mathrm{kg})$ & 19.67 & 6.70 \\
Natural frequency of bounce on front axle $(\mathrm{Hz})$ & 3.52 & 2.49 \\
Natural frequency of bounce on real axle $(\mathrm{Hz})$ & 3.52 & 2.49 \\
\hline
\end{tabular}

\section{Frequency Extraction}

\subsection{Data analysis}

It was expected that the vehicle's dynamic responses would contain bridge vibration characteristics. The authors' first approach was to extract bridge frequencies from the vehicle's dynamic responses. The data analysis procedure was as follows.

The acceleration responses of both trailers were recorded during their passage over the experiment bridge span. A Hanning window was used to remove the end discontinuities from the measured data. For each trailer, the responses of the front and rear axles were averaged to remove 
pitching motions and to extract their pure bounce motions. The processed acceleration response of the front trailer was denoted as "acc1" and that of the rear trailer as "acc2".

To reduce or eliminate the adverse effect of roadway surface profile roughness on the vehicle responses, the method proposed by Yang et al. ${ }^{(13,14)}$ was adopted. First, the Fourier spectra of both trailers' acceleration responses were taken; letting their spectra be denoted as $F S_{a 1}$ and $F S_{a 2}$ for the front and rear trailers respectively. Then, one spectrum was subtracted from the other, yielding a residual spectrum $F S_{\text {sub }}=F S_{a 1}-F S_{a 2}$, which was expected to present bridge-related frequencies if the effect of roadway surface profile roughness is successfully reduced or eliminated. It is noted that, for the purpose of removing these unwanted surface roughness effects from the trailer responses, this subtraction of two spectra would work if both trailers were excited by identical sources (i.e., the roadway surface profile herein), either stationary or nonstationary.

\subsection{Experiment results}

Figure 3 shows a set of the typical acceleration responses $a c c 1$ and $a c c 2$ after synchronization, and Figs. 4(a) and 4(b) show their corresponding Fourier spectra. The speed was $S 1=0.55 \mathrm{~m} / \mathrm{s}$; therefore, it took about $9.5 \mathrm{~s}$ for the vehicle to cross the bridge. From the Fourier spectrum of acc $1\left(F S_{a 1}\right)$, three dominant frequencies were observed: $2.64 \mathrm{~Hz}$, which was close to the trailer's bouncing frequency; $3.32 \mathrm{~Hz}$, which was close to the bridge's first natural frequency i.e., the target frequency; and $5.27 \mathrm{~Hz}$, which might relate to wheel surface roughness (the related theoretical value is $5.84 \mathrm{~Hz}$, calculated by dividing the vehicle's speed, $0.55 \mathrm{~m} / \mathrm{s}$, by the wheel's circumference of $0.094 \mathrm{~m}$ ).

From the Fourier spectrum of acc2 $\left(F S_{a 2}\right)$, one dominant frequency was observed: $5.47 \mathrm{~Hz}$, which might relate to the wheel surface roughness as also observed in $F S_{a 1}$. The two trailers were expected to behave with similar characteristics as discussed in Sect. 2.2. However, owing to different boundary conditions for each trailer, their dynamic characteristics varied. The front trailer was linked with both the tractor ahead and the rear trailer behind, while the rear trailer was linked only with the front trailer ahead. Confined by the two-end links, the front trailer was more stable in both presenting bouncing modes and receiving bridge vibrations than the rear trailer, which was confined at only one end.

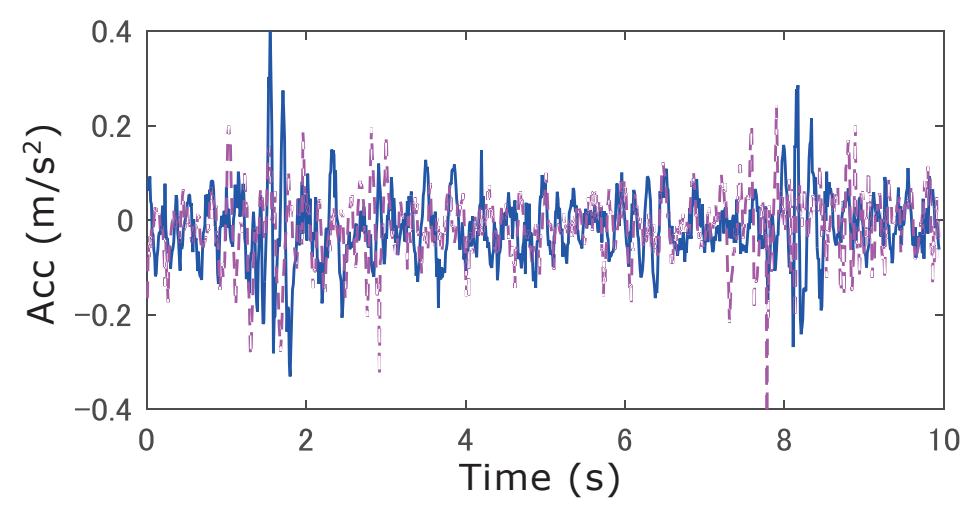

Fig. 3. (Color online) Trailers' acceleration responses acc1 (in pink) and acc2 (in blue), Case $S 1$. 


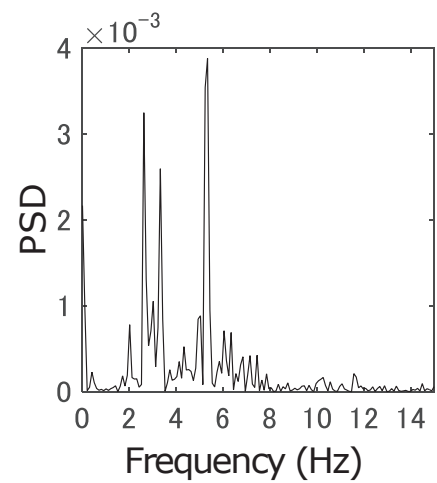

(a)

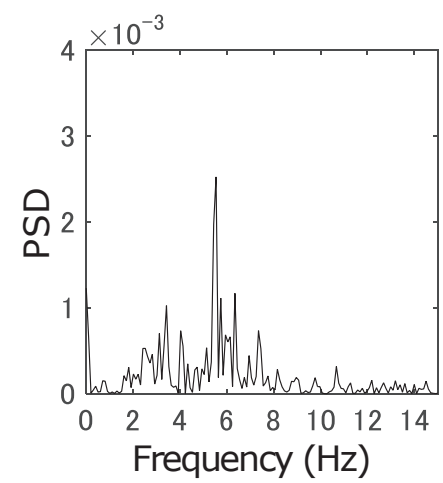

(b)

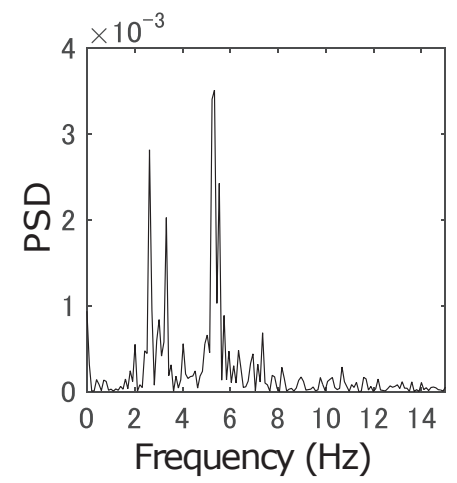

(c)

Fig. 4. Fourier spectra: (a) $F S_{a 1}$, (b) $F S_{a 2}$, and (c) $F S_{\text {sub }}$, Case $S 1$.

The presence of the bridge-related frequency was unexpected, despite a discrepancy of $3.64-3.32$ $=0.32 \mathrm{~Hz}$. This indicates that $F S_{a 1}$ was already suitable for extracting bridge-related frequencies and supports the idea of the drive-by method for extracting bridge frequencies. In contrast, the presence of the trailer's bouncing frequency in the trailer's responses was not surprising, although essentially, it is not the authors' goal to find this frequency. As for the wheel surface-roughnessrelated frequency peak at $5.27 \mathrm{~Hz}$, this does not imply that the surface roughness consisted of a single-frequency component; there were many other components with smaller oscillatory amplitudes as can be observed in both $F S_{a 1}$ and $F S_{a 2}$. Those components unrelated to structural modes were the targets to be reduced or removed in order to improve the visibility of the structural modes.

Subtracting the spectrum of one response from that of the other yields the residual spectrum $F S_{\text {sub }}$, as shown in Fig. 4(c). In the residual spectrum, three dominant frequencies were observed, which were also observed in $F S_{a 1}$ or $F S_{a 2}: 2.64 \mathrm{~Hz}$, which was close to the trailer's bouncing frequency as expected; $3.32 \mathrm{~Hz}$, which is close to the bridge's first natural frequency; and 5.28 $\mathrm{Hz}$, which was regarded to relate to wheel surface roughness. It was observed that the roughnessrelated components were slightly reduced and that few spurious vibration modes were introduced by the subtraction of the two trailers' spectra. The bridge component was also slightly reduced but remained observable. This observation demonstrated that $F S_{\text {sub }}$ would enhance this type of drive-by inspection method.

\section{Damage Detection}

\subsection{Data analysis}

The drive-by method for detecting damage in bridges was also tested. In this laboratory experimental study, the decrease in bridge frequency was considered by attaching several mass blocks beneath the mid-span of the bridge. Four scenarios were considered: each with $0 \mathrm{~kg}$ (denoted as INT), $8.3 \mathrm{~kg}$ (DMG1), $12.8 \mathrm{~kg}$ (DMG2), and $17.2 \mathrm{~kg}$ (DMG3) of attached mass. The first natural frequency and the damping ratio of the bridge for each scenario are summarized in Table 3. It is important to note that the attached blocks were not supposed to resemble any real damage 
Table 3

Damage scenarios and the corresponding attached mass $(m)$, identified first natural frequency $\left(f_{b 1}\right)$, and damping ratio $(\xi)$.

\begin{tabular}{lccc}
\hline Scenario & $m(\mathrm{~kg})$ & $f_{b 1}(\mathrm{~Hz})$ & $\xi$ \\
\hline INT & 0 & 3.64 & 0.011 \\
DMG1 & 8.3 & 3.50 & 0.009 \\
DMG2 & 12.8 & 3.47 & 0.008 \\
DMG3 & 17.2 & 3.42 & 0.007 \\
\hline
\end{tabular}

in practice, but were used to introduce a change in the bridge dynamic properties, which serves as the target for a feasibility study on the drive-by damage detection method. For convenience, the addition of the blocks is still referred to as "damage" here. Moving vehicle tests were conducted for each scenario, consisting of multiple repeated runs over the bridge. For each run, the responses of the two trailers were recorded, processed, and analysed as described in Sect. 3.1. Herein, the speed was kept constant at $S 2(1.05 \mathrm{~m} / \mathrm{s})$.

Damage in the experiment bridge may change the frequency distribution pattern in the residual Fourier spectrum $F S_{\text {sub }}$. It follows that a candidate observation presenting a change in the pattern may indicate damage. Figure 5 presents typical residual Fourier spectra for all the scenarios, where the change in frequency distribution pattern can be observed. Generally, the dominant frequency between 3.0 to $3.5 \mathrm{~Hz}$ (regarded as a bridge-related frequency) shifted leftwards, i.e., decreased as the damage level increased, which agreed with mechanical principles. However, the insufficient accuracy of the frequency identification, caused by the very low spectral resolution, prevented this bridge-related frequency from serving as an effective damage indicator.

Alternatively, the frequency distribution pattern around this target frequency was considered as a damage indicator, which may depend less on spectral resolution. Herein, the change in frequency distribution pattern was evaluated using the Mahalanobis distance (MD), ${ }^{(15)}$ taking the Fourier amplitudes in a certain frequency band as variables and taking the observations from the INT scenario as a reference. Twenty runs of the experiment were conducted for the INT scenario and ten runs each were conducted for the DMG1, DMG2, and DMG3 scenarios.

\subsection{Experiment results}

Figure 6 shows the MDs, taking the amplitudes of $F S_{\text {sub }}$ in the frequency bands of 3.0 to $3.5 \mathrm{~Hz}$ and 3.5 to $4.0 \mathrm{~Hz}$ as variables. These two frequency bands were selected because the above preliminary tests revealed that each band involved the bridge's first natural frequency at a particular damage level. The mean value of MDs was also calculated and plotted for each scenario.

Overall, it was observed that the MDs showed noticeable differences between the experiment scenarios, except for the DMG2 scenario in the 3.5 to $4.0 \mathrm{~Hz}$ band. It is worth noting that the frequency band of 3.0 to $3.5 \mathrm{~Hz}$, which included the bridge-related frequency measured by the drive-by method (3.32 Hz in Sect. 3.2), always showed a clear noticeable difference and hence would be more sensitive to the artificial damage. Also, the MDs increased as the damage level increased, implying that the MDs could indicate not only the presence but also the level of damage. Such observations were encouraging; they implied that the statistical pattern of the distribution of $F S_{\text {sub }}$ could be a damage indicator incorporated in the drive-by bridge inspection. 


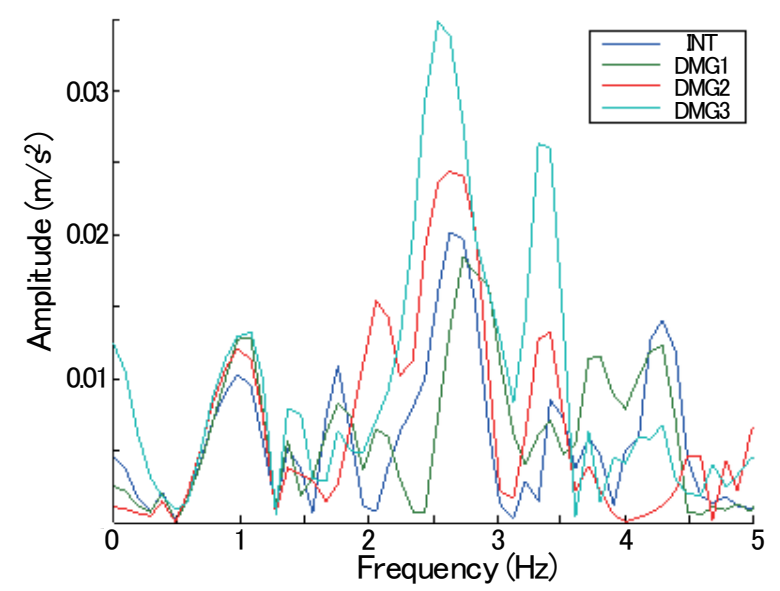

Fig. 5. (Color online) Fourier spectra for all scenarios.

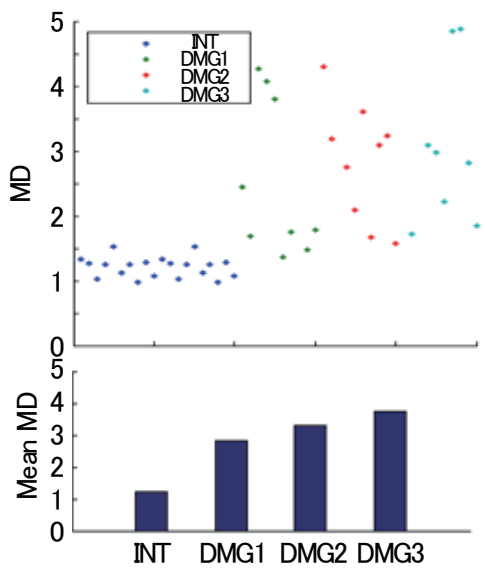

(a)

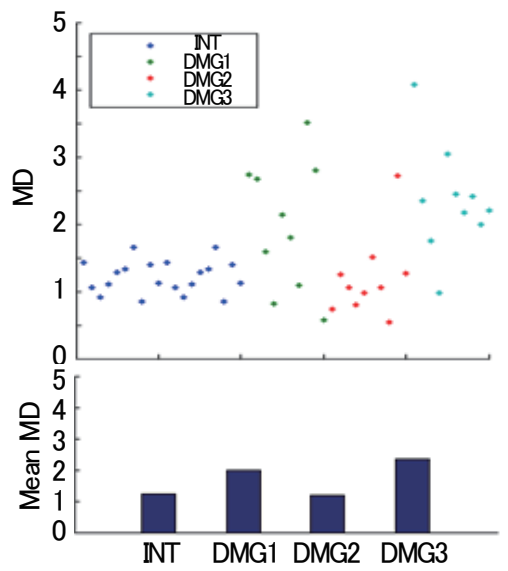

(b)

Fig. 6. (Color online) MDs (top) and their means (bottom): (a) 3.0 to $3.5 \mathrm{~Hz}$ and (b) 3.5 to $4.0 \mathrm{~Hz}$.

\section{Roadway Surface Profile Identification}

\subsection{Data analysis}

Existing research has shown that the roadway surface profile can be identified from vehicle acceleration responses. ${ }^{(9)}$ The identification algorithm developed by McGetrick et al. ${ }^{(9)}$ was adopted here. This algorithm uses a regularized least-squares method to determine a set of roadway surface profiles, which minimizes the discrepancy between calculated and measured vehicle dynamic responses. The numerically calculated vehicle dynamic response was obtained by solving a twodimensional vehicle-bridge interaction problem. This involved a two-axle half-car model, having two degrees of freedom, moving on a finite-element-modelled bridge with a randomly generated roadway surface profile. The measured vehicle dynamic response was that obtained from accelerometer measurements during experimental testing. 
For the roadway surface profile identification experiment, the test vehicle system consisted of a single tractor with no trailer; the tractor part of the tractor-trailer system presented in Sect. 2.2 was used alone herein (the term "tractor" was retained for consistency despite the improper indication of a tractor without a trailer). Observations were obtained from two measurement locations on the vehicle, one being directly above the front suspension component and the other directly above the rear suspension component. The acceleration responses at these locations were measured using accelerometers in the experiment and calculated in the aforementioned finite element simulations. The measured and calculated acceleration responses were then input to the identification algorithm in order to identify the roadway surface profiles.

\subsection{Experiment results}

The identified roadway surface profiles and their power spectral density (PSD) curves were compared with true ones, as shown in Fig. 7. Cases $S 1=0.55 \mathrm{~m} / \mathrm{s}$ and $S 3=2.45 \mathrm{~m} / \mathrm{s}$ were considered herein. For both cases, although the identified surface profile did not always agree with the true profile very well, their relative crests and troughs, i.e., lower frequency components, correspond closely to those of the true profile. This indicated that the spatial frequency distributions would match better than the spatial profiles, as seen in the PSD curves, which are a more appropriate measure of roadway surface profile roughness. This is quite acceptable in view of such an ill-posed inverse problem.
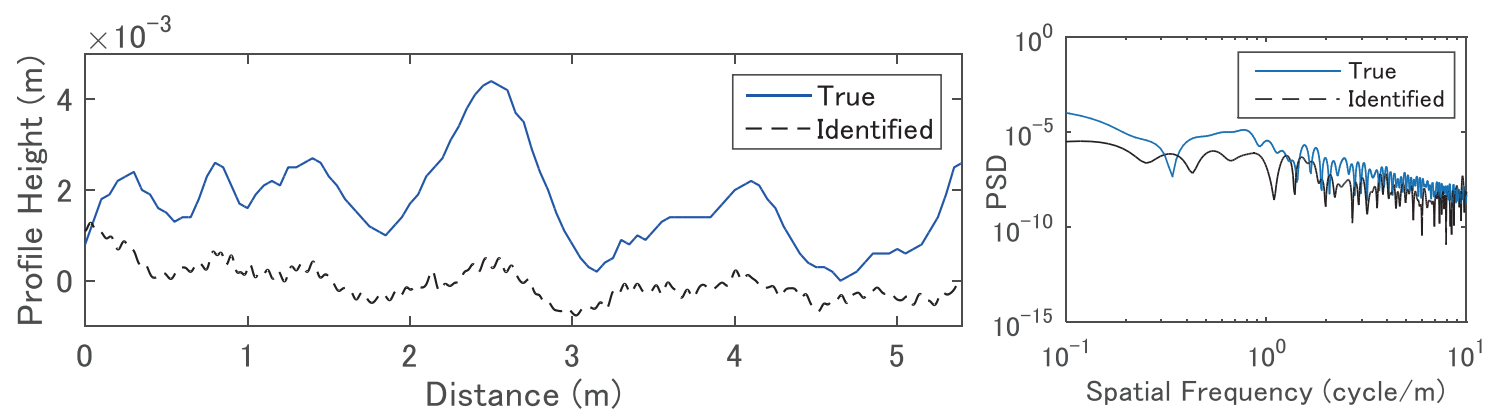

(a)
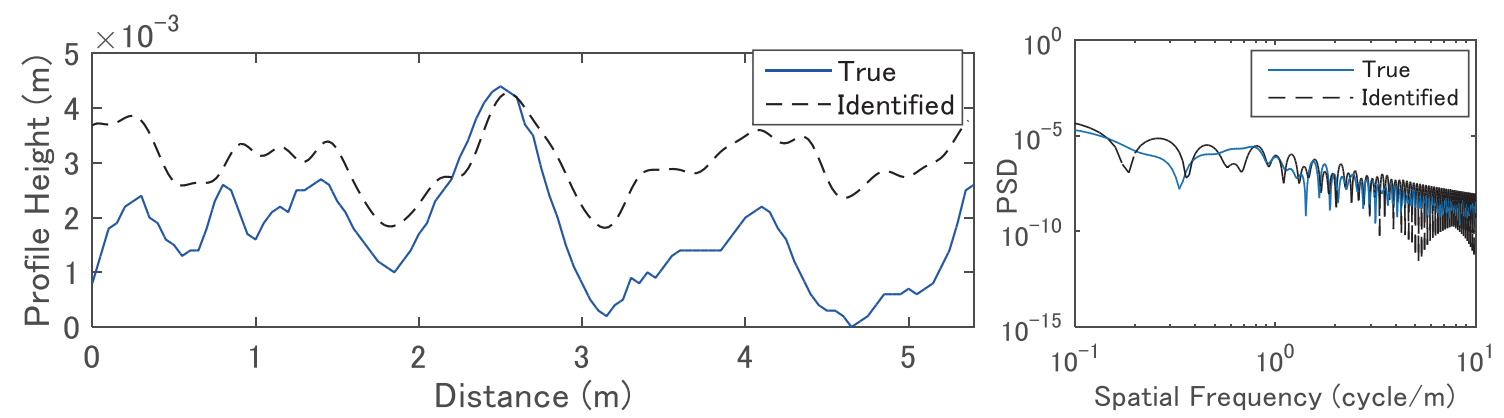

(b)

Fig. 7. (Color online) Identified vs true road surface profiles (left) and their PSDs (right): (a) Case $S 1$ and (b) Case $S 3$. 
The accuracy of the identified roadway surface profiles depended on the speed of the experimental vehicle. As the speed increased from 0.55 to $2.45 \mathrm{~m} / \mathrm{s}$, the identified surface profile agreed better with the true profile, in both spatial and spatial frequency domains. It can be interpreted that a vehicle moving at a higher speed would be excited more easily and predominantly by the roadway surface, and hence the roadway surface profiles, as the vibration source in this inverse dynamic problem, could be identified more accurately. This is a promising result for implementing this method in practice at highway speeds.

\section{Concluding Remarks}

Through laboratory experiments using a test vehicle system equipped with accelerometers, the practical feasibility of the following drive-by bridge condition screening methods was illustrated: (1) bridge-frequency extraction using the Fourier spectrum of a vehicle's dynamic response, (2) damage detection using the change in a vehicle's spectral distribution pattern, and (3) roadway surface profile identification. For tractor-trailer system measurements, in obtaining the residual spectrum by subtracting the spectrum of one trailer from that of the other, it was possible to identify the bridge-related frequencies more effectively. Also, in the residual spectrum, it was shown that the pattern of the frequency distribution may serve as an effective damage indicator, i.e., the change in this pattern may indicate potential damage of the test bridge. Finally, it was shown that a single vehicle equipped with accelerometers could identify the roadway surface profile to an acceptable degree with relatively low frequency components accurately located. These experimental results are valuable for the further development of low-cost and mobile drive-by inspection systems, while field trials will be necessary to support the general applicability of the methods tested in this experimental investigation.

\section{References}

1 Ministry of Land, Infrastructure, Transport and Tourism (MLIT): 2013 Annual Report on Road Statistics: Current State of Bridges (MLIT, Tokyo, 2013).

2 P. Chupanit and C. Phromsorn: Int. Scholarly Sci. Res. Innov. 6 (2012) 389.

3 E. P. Carden and P. Fanning: Struct. Health Monit. 3 (2004) 355.

4 C. R. Farrar, S. W. Doebling, and D. A. Nix: Philos. Trans. R. Soc. London A 359 (2001) 131.

5 Y. B. Yang, C. W. Lin, and J. D. Yau: J. Sound Vib. 272 (2004) 471.

6 C. W. Lin and Y. B. Yang: Eng. Struct. 27 (2005) 1865.

7 C. W. Kim, R. Isemoto, P. J. McGetrick, M. Kawatani, and E. J. Obrien: Smart Struct. Syst. 13 (2014) 775.

8 J. Q. Bu, S. S. Law, and X. Q. Zhu: J. Eng. Mech. ASCE 132 (2006) 1372.

9 P. J. McGetrick, C. W. Kim, A. Gonzalez, and E. J. Obrien: Int. J. Archit. Eng. Constr. 2 (2013) 1.

10 A. Malekjafarian, P. J. McGetrick, and E. J. Obrien: Shock Vib. (2015) ID 286139.

11 F. Cerda, S. Chen, J. Bielak, J. H. Garrett, P. Rizzo, and J. Kovacevic: Smart Struct. Syst. 13 (2014) 849.

12 Y. B. Yang and K. C. Chang: J. Sound Vib. 322 (2009) 718.

13 Y. B. Yang, Y. C. Li, and K. C. Chang: Interact. Multiscale Mech. 5 (2012) 347.

14 Y. B. Yang, Y. C. Li, and K. C. Chang: Acta Mech. 223 (2012) 1851.

15 G. Taguchi and R. Jugulum: The Mahalanobis—Taguchi Strategy_A Pattern Technology System (Wiley, NY, 2002). 


\section{About the Authors}
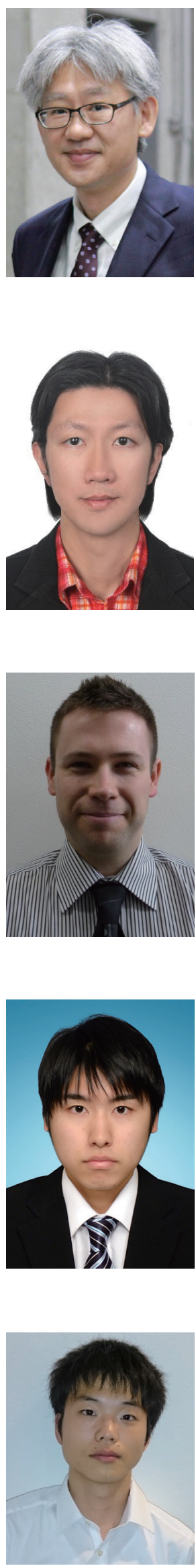

Chul-Woo Kim received his Dr. Eng. degree from Kobe University, Japan, in 2003. He has been a professor at Kyoto University since 2009. His main area of research is related to bridge dynamics, direct monitoring, smart sensors for infrastructure, reliability and optimization of structural systems, vibrations, and seismic responses. In addition to his research, he consults with municipalities both in Japan and abroad about managing their infrastructure.

Kai-Chun Chang received his Ph.D. degree from National Taiwan University, Taiwan, in 2009. From 2009 to 2011, he was a postdoctoral researcher at the same university. From 2011 to 2015, he was a visiting researcher and Specified Assistant Professor at Kyoto University, Japan. Since 2015, he has been a Junior Associate Professor at the same university. His research interests are in bridge structural dynamics and vibration-based damage detection.

Patrick McGetrick received his Ph.D. degree from University College Dublin, Ireland in 2012. From 2012 to 2014, he was an Assistant Professor at Kyoto University, Japan. Since 2014, he has been a Lecturer at Queen's University Belfast, Northern Ireland, UK. His research interests are oriented toward the application of sensors and vehicle-bridge interaction systems to bridge monitoring, structural damage detection, and road profile identification.

Shinichi Inoue received his Master's degree from Kyoto University, Japan, in 2016. He was involved in the study on a drive-by bridge inspection for his master's research. Since 2016, he has been an engineer at Nikken Sekkei Civil Engineering Ltd., Japan, primarily in charge of plant structural design.

Souichirou Hasegawa received his undergraduate degree from Kyoto University, Japan in 2016. Since 2016, he has been a graduate student at Kyoto University, Japan. His research interests are in bridge monitoring and road profile identification utilizing moving vehicles. 